ARTICLE

\title{
Direct observation of multistep energy transfer in LHCll with fifth-order 3D electronic spectroscopy
}

Zhengyang Zhang ${ }^{1}$, Petar H. Lambrev², Kym L. Wells ${ }^{1}$, Győző Garab² \& Howe-Siang Tan ${ }^{1}$

During photosynthesis, sunlight is efficiently captured by light-harvesting complexes, and the excitation energy is then funneled towards the reaction centre. These photosynthetic excitation energy transfer (EET) pathways are complex and proceed in a multistep fashion. Ultrafast two-dimensional electronic spectroscopy (2DES) is an important tool to study EET processes in photosynthetic complexes. However, the multistep EET processes can only be indirectly inferred by correlating different cross peaks from a series of 2DES spectra. Here we directly observe multistep EET processes in LHCII using ultrafast fifth-order three-dimensional electronic spectroscopy (3DES). We measure cross peaks in 3DES spectra of LHCll that directly indicate energy transfer from excitons in the chlorophyll $b(\mathrm{Chl} b$ ) manifold to the low-energy level chlorophyll $a(\mathrm{Chl} a)$ via mid-level $\mathrm{Chl} a$ energy states. This new spectroscopic technique allows scientists to move a step towards mapping the complete complex EET processes in photosynthetic systems.

\footnotetext{
${ }^{1}$ Division of Chemistry and Biological Chemistry, School of Physical and Mathematical Sciences, Nanyang Technological University, 21 Nanyang Link, Singapore 637371. ${ }^{2}$ Biological Research Centre, Hungarian Academy of Sciences, Temesvári krt. 62, Szeged 6726, Hungary. Correspondence and requests for materials should be addressed to H.-S.T. (email: howesiang@ntu.edu.sg).
} 
$2 \mathrm{D}$ electronic spectrum is presented in two frequency dimensions, which can be termed the excitation frequency and the emission frequency. Two-dimensional electronic spectroscopy (2DES) $)^{1-3}$ measures a two-point correlation plot, and is an excellent tool to measure single-step excitation energy transfer (EET) processes as the appearance and evolution of offdiagonal cross peaks provide a direct observation of electronic coupling between the donor and acceptor pigment molecules, by reading off the corresponding frequencies on the excitation and emission axes, respectively ${ }^{4-7}$. However, multistep EET processes are only indirectly inferred from the 2DES measurements ${ }^{8}$. Since it is clear that complex multistep EET network is present in most light-harvesting complexes ${ }^{9-12}$, higher-order multidimensional electronic spectroscopy will be needed to directly measure multistep EET processes.

Fifth-order 3D optical spectroscopy is an extension of thirdorder 2D optical spectroscopy ${ }^{13-18}$. Applications include the probing of higher states of vibrational systems in order to better characterize the anharmonicity 14,19 , the testing of nonMarkovian processes ${ }^{20}$ and the characterizing of electron transfer systems $^{21}$

In fifth-order 3D optical spectroscopy, a spectrum $S^{(5)}\left(\omega_{1}, \omega_{3}\right.$, $\left.\omega_{5} ; t_{2}, t_{4}\right)$ with three frequency axes parametrized with two population times $t_{2}$ and $t_{4}$, is measured. A two-step EET process that proceeds from state $\mathrm{A}$ to state $\mathrm{B}$ over time $t_{2}$ and from state $\mathrm{B}$ to state $\mathrm{C}$ over time $t_{4}$ results in the emergence of a $3 \mathrm{D}$ cross peak $\left(\omega_{1}, \omega_{3}, \omega_{5}\right)=\left(\omega_{A}, \omega_{B}, \omega_{C}\right)$ on a $3 D$ spectrum, where $\omega_{A}, \omega_{B}$ and $\omega_{C}$ are the absorption frequencies of state $A, B$ and $C$, respectively. In comparison, a conventional $2 \mathrm{D}$ spectrum is presented as $S^{(3)}$ $\left(\omega_{1}, \omega_{3} ; t_{2}\right)$ with a population time $t_{2}$. In addition, if the conventional 2D spectrum $S^{(3)}\left(\omega_{1}, \omega_{3} ; t_{2}\right)$ is measured for a series of population times $t_{2}$ and Fourier transformed about $t_{2}$, one can obtain a data set $S^{(3)}\left(\omega_{1}, \omega_{3} ; \omega_{2}\right)$. As there are three frequency axes involved, it has been commonly termed $3 \mathrm{D}$ spectroscopy as well $^{22,23}$. This third-order 3D optical spectroscopy $S^{(3)}\left(\omega_{1}, \omega_{3} ; \omega_{2}\right)$ is different from the fifth-order $3 \mathrm{D}$ optical spectroscopy $S^{(5)}\left(\omega_{1}, \omega_{3}, \omega_{5} ; t_{2}, t_{4}\right)$, which is the subject of this article.

There are several other fifth-order multipulse nonlinear optical experiments such as the family of pump-dump-probe (PDP) and pump-repump-probe spectroscopies ${ }^{24}$ and the multiple population-period transient spectroscopy ${ }^{25}$. In these experiments, the system interacts at two moments in time (separated by a first population period) with the light field. This is followed by a second population period before a transient absorption is obtained. Therefore these experiments give a transient spectrum as a function of the two population time $S^{(5)}\left(\omega_{5} ; t_{2}, t_{4}\right)$. There is no frequency resolution (apart from the coarse resolution determined by the laser-pulse spectral bandwidth) for the two interactions. The fifth-order $3 \mathrm{D}$ optical spectroscopy described in this paper can be viewed as a general form of these fifth-order experiments. In addition to the two controllable population periods, fifth-order 3D optical spectroscopy has three coherence periods that give rise to three frequency dimensions. This gives the three-point frequencyfrequency correlation as a function of two population times $S^{(5)}\left(\omega_{1}, \omega_{3}, \omega_{5} ; t_{2}, t_{4}\right)$. Another kind of fifth-order optical experiment, 2D Raman spectroscopy was plagued with spurious signal arising from third-order cascading processes ${ }^{26}$. It has been shown in control tests performed for fifth-order resonant optical experiments (such as the fifth-order 3D electronic spectroscopy experiments discussed in this article) that there is no significant contamination from third-order cascading signals ${ }^{15,19,27}$. If for some systems or conditions that the cascading signals become significant, procedures are available to isolate the contaminant signals ${ }^{28}$.

A fifth-order 3D optical spectroscopy experiment involves the control of up to six (including local oscillator pulse) phase-locked pulses and is extremely challenging. Purely absorptive fifth-order 3D optical spectroscopy was first demonstrated in the midinfrared $^{29}$ and only recently did we first demonstrate a purely absorptive fifth-order 3D electronic spectroscopy in the visible (three-dimensional electronic spectroscopy (3DES)) based on a pulse shaping pump-probe setup ${ }^{17,27}$.

Plant light-harvesting complex II (LHCII) - the most abundant membrane protein in nature, is responsible for the absorption of about half of the radiation used for plant photosynthesis and plays key roles in regulating the energy input to Photosystem II to optimize photosynthetic efficiency while avoiding damage ${ }^{30,31}$.

In this article, we report the use of 3DES to directly observe two-step EET processes in LHCII. We observe 3D cross peaks that represent a two-step EET process from the chlorophyll $b$ (Chl $b$ ) manifold to the low-energy level chlorophyll $a(\mathrm{Chl} a)$ via mid-level Chl $a$ energy states. The population time dependence of the $3 \mathrm{D}$ cross peak amplitude corresponds well with corresponding 2DES measurements. We provide possible assignments of the observed spectral features based on published structure-based models.

\section{Results}

Fifth-order 3DES. In fifth-order 3DES, five laser pulses, with inter-pulse delay times $t_{1}, t_{2}, t_{3}$ and $t_{4}$, interact with the sample and a fifth-order optical signal is emitted at time $t_{5}$. The pulse sequence scheme is shown in Fig. 1a. The periods $t_{1}, t_{3}$ and $t_{5}$ are the coherence times where the excitons oscillate at their transition frequencies. The periods $t_{2}$ and $t_{4}$ are the population times, during which the population transfers between different excitons. The final coherence over $t_{5}$ results in an optical signal that is emitted in a phase-matched direction and can be measured by frequency-resolved heterodyne detection (along the $\omega_{5}$ axis). At given population times $t_{2}$ and $t_{4}$, the experiment is scanned over the coherence periods $t_{1}$ and $t_{3}$. The data are processed and then Fourier transformed about coherence times $t_{1}$ and $t_{3}$ to obtain a 3D spectrum $S^{(5)}\left(\omega_{1}, \omega_{3}, \omega_{5} ; t_{2}, t_{4}\right)$. The $3 \mathrm{D}$ cross peak feature that represents a two-step EET process that proceeds from state A to state $\mathrm{B}$ over time $t_{2}$ and from state $\mathrm{B}$ to state $\mathrm{C}$ over time $t_{4}$, can be outlined using the Liouville pathways diagrams (double-sided Feynmann diagrams $)^{32}$ in Fig. 1b. The labels for the processes $R_{1}$, $R_{2}, R_{3}$ and $R_{4}$ follow the convention of Hamm ${ }^{13}$. The shaded areas denote the population periods where EET processes proceed between the states separated by the dashed lines.

Linear spectroscopy and 2DES of LHCII. According to the crystallographic structure of $\mathrm{LHCII}^{33}$, each monomer in the trimeric pigment-protein complex binds 14 chlorophylls (8 Chl $a$ and $6 \mathrm{Chl} b^{33}$ ). The 14 excitons are closely spaced, which makes it hard to elucidate the different timescales of every energy migration dynamics in the multistep EET processes. Henceforth we will adopt the more often used wavelength unit, $\lambda$ instead of the frequency unit $\omega$. The linear absorption spectrum (Fig. 2a) can be divided into three regions: high-energy exciton region $\left(\lambda_{\mathrm{H}}<660 \mathrm{~nm}\right)$, encompassing transitions to Chl $b$ and high-energy $\mathrm{Chl} a$ exciton states, the mid-energy region $\left(\lambda_{M}=660-675 \mathrm{~nm}\right)$, which contains mid-energy Chl a states, and the low-energy exciton region $\left(\lambda_{\mathrm{L}}>675 \mathrm{~nm}\right)$, which contains the lowest-energy Chl $a$ exciton states. In the following, we will demonstrate how $3 \mathrm{DES}$ can be employed to observe the multistep flow of energy between the three wavelength regions $\lambda_{\mathrm{H}} \rightarrow \lambda_{\mathrm{M}} \rightarrow \lambda_{\mathrm{L}}$.

As a reference, 2DES of the EET process was performed as well, and a representative $2 \mathrm{D}$ spectrum at population time $t_{2}=300 \mathrm{fs}$ is shown in Fig. 2b. More 2D spectra at various $t_{2}$ have been included in Supplementary Fig. 1. Two cross peak regions, 
a
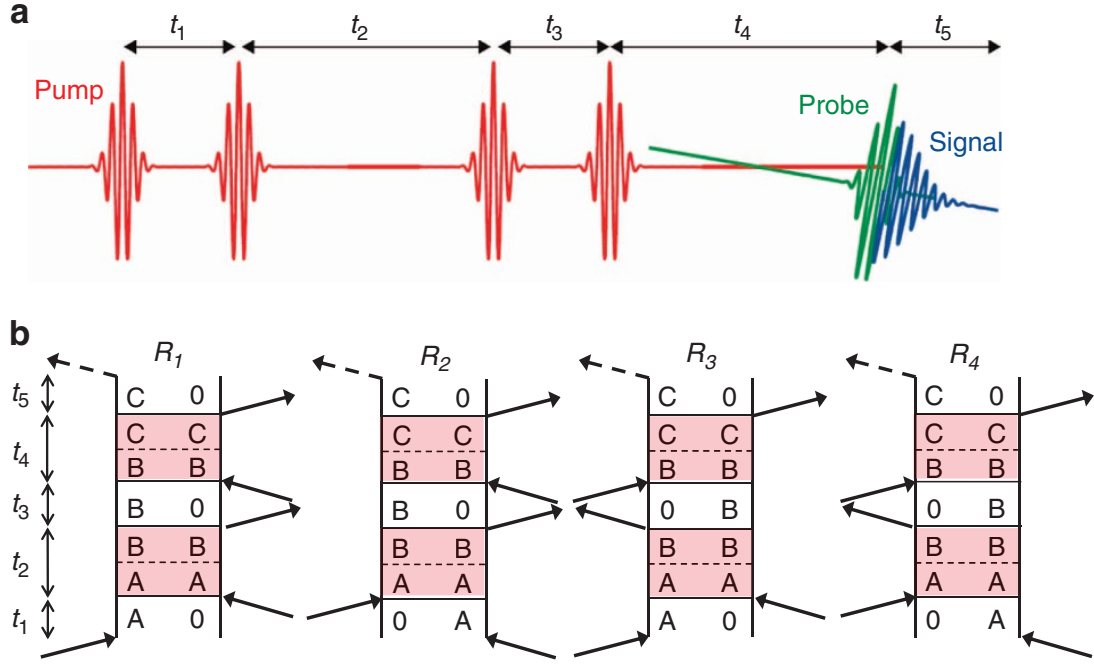

Figure 1 | Three-dimensional electronic spectroscopy (3DES) pulse sequence and Liouville pathways diagrams. (a) Pulse sequence used in coherent fifth-order 3D optical spectroscopy performed in a pulse-shaper assisted pump-probe geometry. The pulse-shaper creates a four-pulse pump sequence (red) with controllable delays and relative phases followed by the probe (green) that interacts at a small angle to emit the signal (blue). (b) A 3D cross peak $\left(\omega_{1}, \omega_{3}, \omega_{5}\right)=\left(\omega_{A}, \omega_{B}, \omega_{C}\right)$ on a 3DES spectrum is a combination of fifth-order optical processes outlined in the Liouville pathways (double-sided Feynman diagrams) depicted above ${ }^{16}$. The shaded areas denote the population periods where EET processes proceed between the states separated by the dashed lines. The label of the processes $R_{1}, R_{2}, R_{3}$ and $R_{4}$ follows the convention of Hamm ${ }^{10}$.

$\mathrm{H} / \mathrm{L}$ and $\mathrm{H} / \mathrm{M}$, are labelled in Fig. $2 \mathrm{~b}$, which is ascribed to EET from the high-energy exciton region to the low-energy region $\left(\lambda_{H} \rightarrow \lambda_{L}\right)$ and to the mid-energy region $\left(\lambda_{H} \rightarrow \lambda_{M}\right)$, respectively. The cross-peak amplitudes at $\mathrm{H} / \mathrm{L}(655 \rightarrow 678 \mathrm{~nm})$ and $\mathrm{H} / \mathrm{M}$ $(655 \rightarrow 670 \mathrm{~nm})$ are plotted against $t_{2}$ in Fig. 2c. The H/M amplitude initially rises rapidly on a timescale of $\sim 300 \mathrm{fs}$ due to EET from Chls $b$ to mid-energy Chls $a$. The H/M cross peak subsequently decays on a longer timescale of $\sim 2 \mathrm{ps}$, which is a result of EET out of the mid-energy $\mathrm{Chl} a$ region. The H/L cross peak amplitude shows only an increasing trend. Apart from indicating the direct $\lambda_{\mathrm{H}} \rightarrow \lambda_{\mathrm{L}}$ transfer, a multistep EET process $\lambda_{\mathrm{H}} \rightarrow \lambda_{\mathrm{M}} \rightarrow \lambda_{\mathrm{L}}$ can be indirectly inferred from the correlated timescale of the decay of the H/M peak and the rise of the H/L cross peak, as it has been previously reported ${ }^{8,34}$. It is evident that the cross peaks may not be readily resolved in the $2 \mathrm{D}$ spectra, due to the broad inhomogeneous widths of the spectral features. In this regard, 3DES has the advantage of being able to spectrally resolve closely spaced or overlapping exciton bands in the additional frequency axis $\omega_{5}$, and also by correlating energy transfers with the three frequency axes.

Fifth-order 3DES of LHCII. In the 3D spectra of LHCII presented in this article, the population time $t_{2}$ was kept fixed at $300 \mathrm{fs}$, and spectra were taken at different population times $t_{4}$ from 150 fs to $8 \mathrm{ps}$. A graphical representation of the $3 \mathrm{D}$ spectrum at $t_{2}=300 \mathrm{fs}, t_{4}=800 \mathrm{fs}$ is shown in Fig. 3. The rendered isosurface represents amplitude values of 0.1 relative to the global maximum. The maximum along $\lambda_{1}$ and $\lambda_{3}$ is found near the excitation wavelength, $668 \mathrm{~nm}$; the peak along the detection wavelength $\lambda_{5}$ coincides with the pump-probe maximum, $678 \mathrm{~nm}$. A cutaway is made at $\lambda_{5}=682 \mathrm{~nm}$ to better illustrate the features and structure of the $3 \mathrm{D}$ spectra. The amplitude of the $3 \mathrm{D}$ spectrum on this cutaway slice is colour coded. The amplitude is concentrated on the $\lambda_{3}=\lambda_{1}$ diagonal plane, representing all the states whose population has not decayed during $t_{2}$. It is evident from the figure that the $3 \mathrm{D}$ spectrum features additional crosscorrelation amplitudes off the $\lambda_{3}=\lambda_{1}$ diagonal plane, particularly in the region $\lambda_{3}<\lambda_{1}$. This indicates downhill energy transfer during the population time $t_{2}$. One prominent feature to note is a ridge (or $3 \mathrm{D}$ cross peak) along the $\lambda_{5}$ axis, around $\lambda_{1}=655 \mathrm{~nm}$, $\lambda_{3}=670 \mathrm{~nm}$. The ridge's shape and feature evolves with the different population times $t_{4}$ in our experiments and is the main subject of our discussion below. For comparison, representative $3 \mathrm{D}$ spectra at $t_{2}=300 \mathrm{fs}$ and $t_{4}=150 \mathrm{fs}$ and $5 \mathrm{ps}$ are presented in Supplementary Fig. 2. Qualitatively, one can discern the evolution of the $3 \mathrm{D}$ cross peaks clearly with different delays $t_{4}$.

To better visualize the time evolution of the $3 \mathrm{D}$ cross peaks, slices of the $3 \mathrm{D}$ spectra at wavelengths $\lambda_{1}=655 \mathrm{~nm}$ and $\lambda_{1}=665$ $\mathrm{nm}$ for three different population times $t_{4}$ are shown as quasi-2D spectra with wavelength axes $\lambda_{3}$ and $\lambda_{5}$ (Fig. 4). The spectra demonstrate the potential of $3 \mathrm{DES}$ to selectively probe EET pathways with high resolving power. The slices at $\lambda_{1}=655 \mathrm{~nm}$ feature two well-separated cross peaks, at $\lambda_{3} \sim 655 \mathrm{~nm}$ and $\lambda_{3} \sim 670 \mathrm{~nm}$, whereas the slices at $\lambda_{1}=665 \mathrm{~nm}$ have only one band around $665 \mathrm{~nm}$. The most significant feature on the presented slices is the $3 \mathrm{D}$ cross peak $655 \rightarrow 670 \rightarrow 678 \mathrm{~nm}$, which can be denoted $\mathrm{H} / \mathrm{M} / \mathrm{L}$, and interpreted as follows. In a $2 \mathrm{D}$ spectrum at population time $t_{2}$, the $\mathrm{H} / \mathrm{L}$ cross peak amplitude is the conditional population of state $L$ (absorbing at $\lambda_{L}$ ) after population time $t_{2}$, given that the initial excitation is at $\mathrm{H}$ (absorbing at $\lambda_{\mathrm{H}}$ ). Extending the interpretation to 3DES, the 3D cross peak amplitude at $\mathrm{H} / \mathrm{M} / \mathrm{L}$ is the conditional population of $\mathrm{L}$ after population time $t_{2}+t_{4}$ given that (i) the initial excitation is at $\mathrm{H}\left(\lambda_{\mathrm{H}} \sim 655 \mathrm{~nm}\right)$ and (ii) the population after time $t_{2}$ is at $\mathrm{M}$ $\left(\lambda_{M} \sim 670 \mathrm{~nm}\right)$. This gives a direct measurement of a two-step EET process between three different states: $\mathrm{H} \rightarrow \mathrm{M} \rightarrow \mathrm{L}$. The other features of interest in the spectra are along $\lambda_{1}=655 \mathrm{~nm}$, that is, on the diagonal plane $\lambda_{3}=\lambda_{1}$, denoting EET from states $\mathrm{H}$ to states $\mathrm{L}(\mathrm{H} / \mathrm{H} / \mathrm{L}$ cross peak) and from $\mathrm{H}$ to $\mathrm{M}(\mathrm{H} / \mathrm{H} / \mathrm{M}$ cross peak).

Owing to the separation over a third frequency (wavelength) dimension, the spectral regions $H$ and $M$ are fully resolved in the $3 \mathrm{D}$ spectra, whereas in the $2 \mathrm{D}$ spectra (Fig. 2b) they overlap. Moreover, by virtue of using two population times, the kinetics of the direct $\mathrm{H} \rightarrow \mathrm{L}$ and multistep $\mathrm{H} \rightarrow \mathrm{M} \rightarrow \mathrm{L}$ pathways can be unambiguously resolved. The rise dynamics of the $\mathrm{H} / \mathrm{H} / \mathrm{L}$ cross peak amplitude depends solely on the $\mathrm{H} \rightarrow \mathrm{L}$ process, and the rise 
a
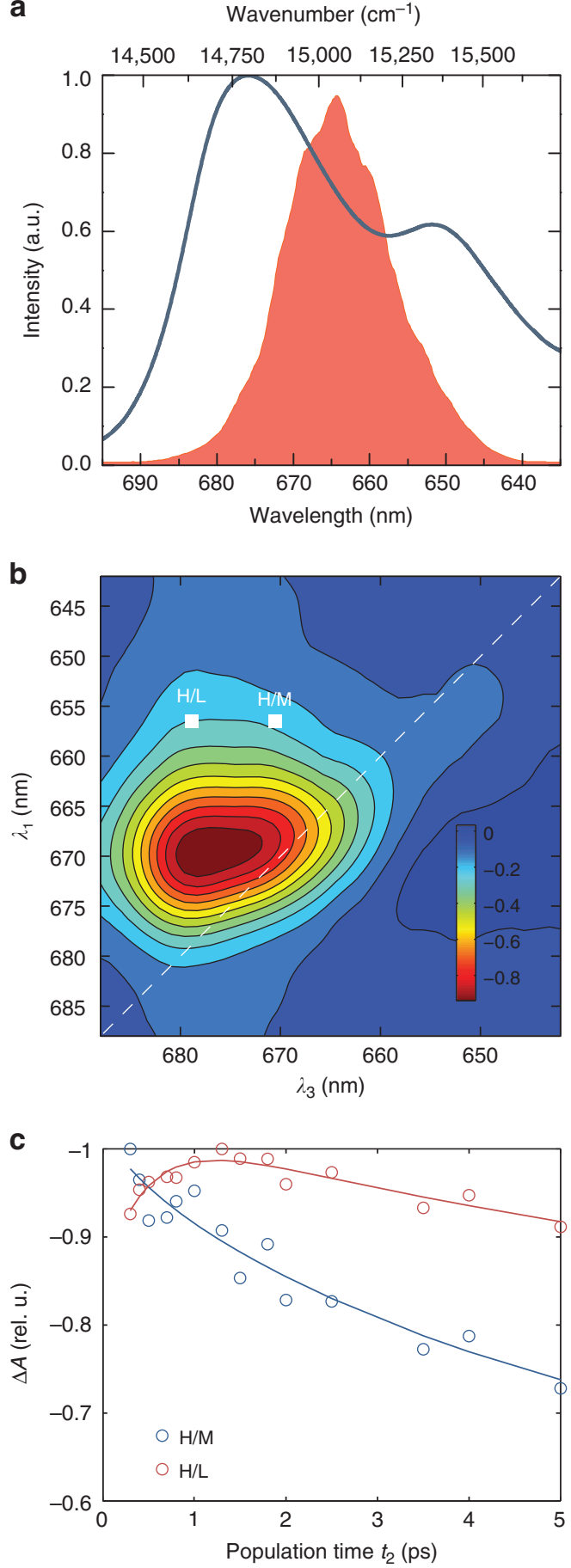

Figure 2 | Linear and 2DES spectrum of LHCII. (a) The experimental linear absorption spectrum of LHCII (solid blue) and the excitation pump spectrum (solid filled pink). (b) Two-dimensional spectrum of LHCII at population time $t_{2}=0.3 \mathrm{ps}$, with the white line indicating the diagonal of the spectra. (c) The amplitude evolution of the integrated exciton cross peaks $657 \rightarrow 670 \mathrm{~nm}$ and $657 \rightarrow 678 \mathrm{~nm}$ as a function of population time $t_{2}$. A multistep EET process can be inferred from the associated decay of the $657 \rightarrow 670 \mathrm{~nm}$ cross peak and the rise of the $657 \rightarrow 678 \mathrm{~nm}$ cross peak.

of the $\mathrm{H} / \mathrm{M} / \mathrm{L}$ peak depends solely on the $\mathrm{M} \rightarrow \mathrm{L}$ process, allowing us, in principle, to determine the respective rate constants directly from the experimental data without the need for soft modelling and the inherent uncertainty associated with it. Figure 5 plots the

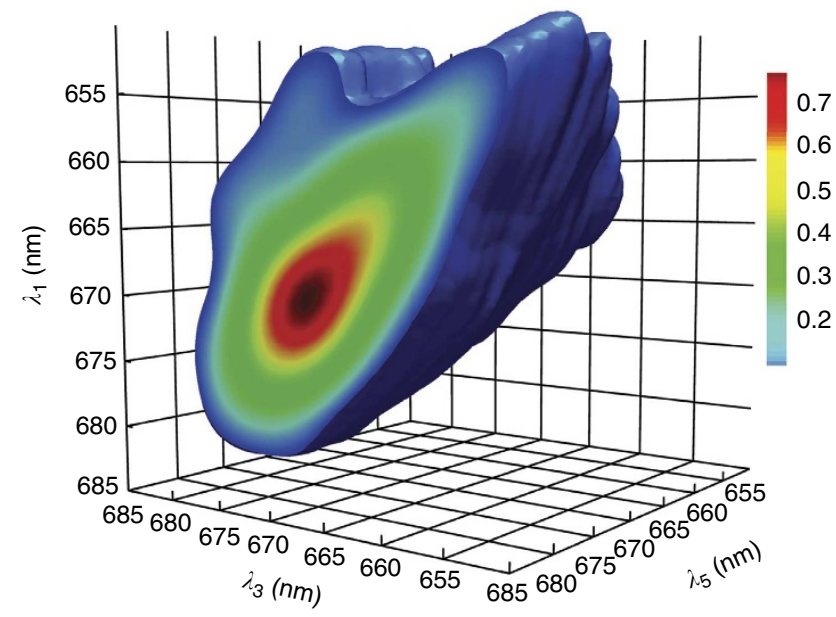

Figure 3 | Three-dimensional electronic spectroscopy (3DES) spectrum of LHCII. Spectrum recorded at population times $t_{2}=0.3 \mathrm{ps}$ and $t_{4}=800 \mathrm{fs}$. The isosurface represents amplitude values of 0.1 relative to the global maximum. The cutaway at $\lambda_{5}=685 \mathrm{~nm}$ to better illustrate the features and structure of the 3D spectra. The prominent feature to note is the ridge along the $\lambda_{5}$ axis, around $\lambda_{1}=655, \lambda_{3}=670$. The ridge's shape and feature evolves with the different population times $t_{4}$ in our experiments.

experimental amplitudes of the $\mathrm{H} / \mathrm{M} / \mathrm{L}$ cross peak as a function of population times $t_{4}$. We simulate the H/M/L cross peak amplitudes by solving a rate equation for a system comprising H, M and L states (Supplementary Note 2). As parameters for the simulations, we use the EET transfer timescales values that have been recently obtained for LHC II trimers ${ }^{34}$, where the Chl $b$ excitonic states (associated with the $\mathrm{H}$ states here) transfer energy to Chl $a$ states (associated with the $\mathrm{M}$ and $\mathrm{L}$ states here) at a timescale of $300 \mathrm{fs}$ and the intermediate Chl $a$ states (associated with the M states here) to low-energy Chl $a$ states (associated with the $\mathrm{L}$ states here) at a $2.3 \mathrm{ps}$ timescale. There is then a $>20 \mathrm{ps}$ relaxation to ground state $\mathrm{g}$. The simulated values (red line in Fig. 5) are consistent with the trend of the experimental values, as can be seen in Fig. 5. The line essentially traces a 2.3 ps timescale rise that represents the $\mathrm{M} \rightarrow \mathrm{L}$ transfer and a subsequent $>20 \mathrm{ps}$ decay representing the decay to ground state.

\section{Discussion}

Assignment of spectral features to structural moieties in the pigment-protein complex is possible by use of structure-based models of the exciton energies and transfer kinetics $9,10,12,35,36$. On the basis of more recent model by Müh et al. ${ }^{36}$, the $\mathrm{H}$ state $\left(\lambda_{\mathrm{H}} \sim 655 \mathrm{~nm}\right)$ can be contributed by either Chl $608(\sim 658 \mathrm{~nm})$ in the stromal layer of Chls $b$, or Chl $607(\sim 654 \mathrm{~nm})$, in the lumenal $\mathrm{Chl} b$ cluster. Although both states are within the relevant wavelength, Chl 607 is incompatible with the observed $\mathrm{H} / \mathrm{H} / \mathrm{L}$ cross peak at $t_{2}=300 \mathrm{fs}$. EET from this $\mathrm{Chl} b$, on the lumenal side, to the L state, which is identified with the Chl 610/ $611 / 612$ cluster on the stromal side, must occur over a much longer time scale. In contrast, rapid EET ( $\leq 1 \mathrm{ps)}$ from Chl 608/ 609 to the $610 / 611 / 612$ cluster, is expected by the structural models of Novoderezhkin et. al. ${ }^{10,12}$, which is in line with the dynamics of the $3 \mathrm{D}$ cross peak. As to the identity of the $\mathrm{M}$ state $\left(\lambda_{M} \sim 670 \mathrm{~nm}\right)$, several pigments may contribute to this spectral region but only the Chl 602/603 pair (also according to Novoderezhkin et $a l .{ }^{10}$ ) would have the longer lifetime compatible with the $3 \mathrm{DES}$ data. The available structural models 

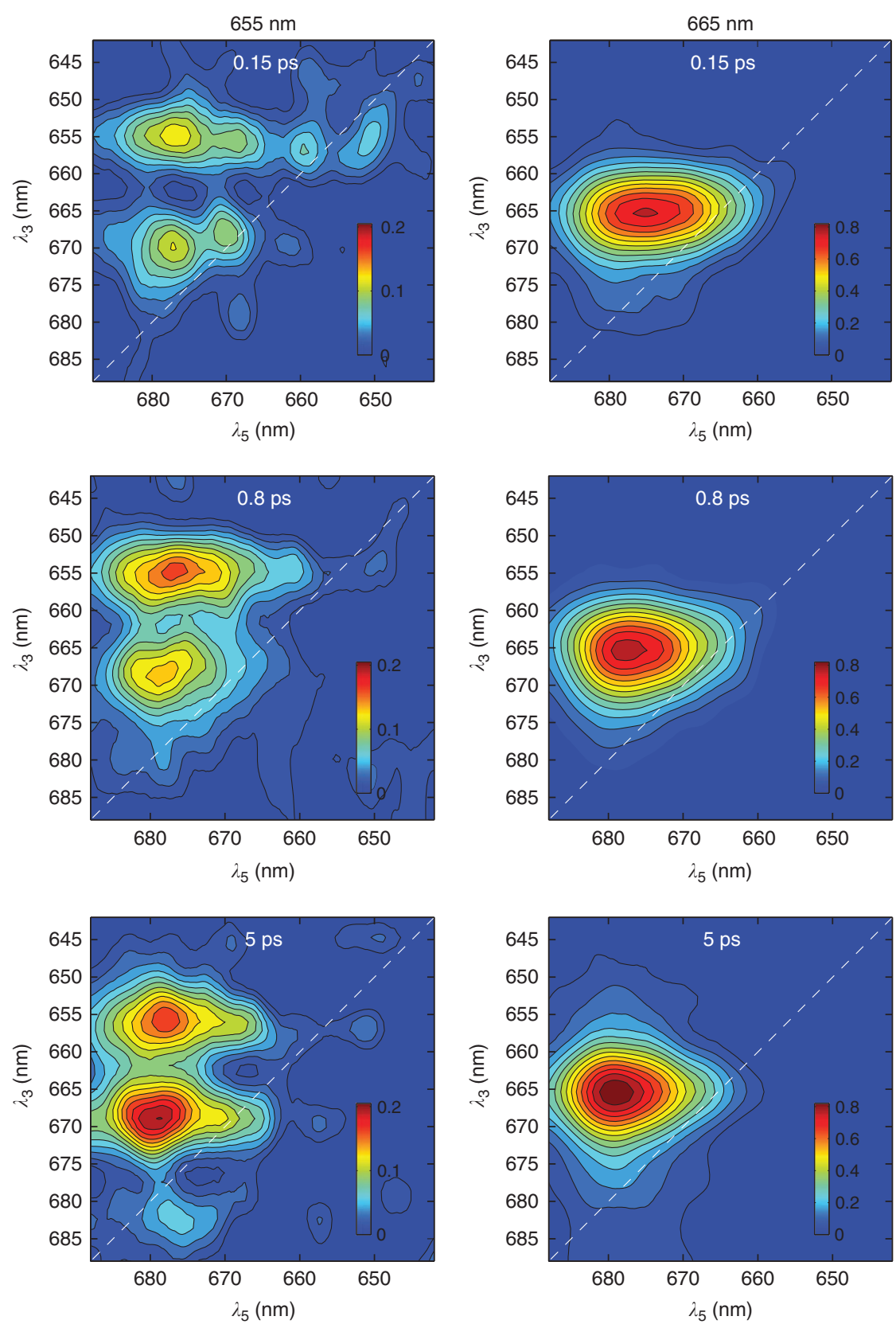

Figure 4 | Two-dimensional slices of 3DES spectra. Slices of the 3D spectra of LHCII at $\lambda_{1}=655 \mathrm{~nm}$ and $\lambda_{1}=665 \mathrm{~nm}$ to obtain quasi-2D spectra, with selected quasi-2D spectra shown at population times $t_{2}=0.3 \mathrm{ps}$ and $t_{4}=0.2,0.8$ and $5 \mathrm{ps}$ (indicated on the plots). The colour scale represents amplitudes relative to the respective 3D spectrum's global maximum.

provide different time constants for EET depending on the chosen theoretical formalism. The EET from Chl 602/603 to the lowest-energy Chls is estimated to be from subpicoseconds ${ }^{10}$ to tens of picoseconds ${ }^{12}$. The kinetics of the H/M/L peak in our experiment is consistent with the rate constant of $\mathrm{M} \rightarrow \mathrm{L}$ transfer between 2 and $6 \mathrm{ps}$ which falls closer to the more recent calculation by Renger et al. ${ }^{12}$

The results presented in this report demonstrate for the first time that direct observation of multistep EET processes in LHCII can be achieved using 3DES. The method allows us to selectively probe EET between single exciton states with defined transition energies, isolated from the congested spectrum of the exciton network. In principle, with two independent population times, the two rate constants of a two-step EET process can be directly obtained from experimental data by measuring the population time-dependent amplitudes of appropriate 3D cross peaks. Fifthorder 3DES represents a valuable extension to $2 \mathrm{D}$ electronic spectroscopy, which is rapidly becoming a standard tool in photosynthesis research, and will undoubtedly help in unravelling the complex energy transfer processes in biological lightharvesting systems. As a much longer experimental time needed to collect a 3D spectrum compared with a 2D spectrum, it may not be practical to obtain 3D spectra for an exhaustive range of population times $t_{2}$ and $t_{4}$. It is envisioned that $2 \mathrm{DES}$ will first be deployed to obtain as much information as possible about the EET rates. 3DES can then be performed for selected population times $t_{2}$ and $t_{4}$ to resolve specific exciton states involved in the multistep EET processes. 


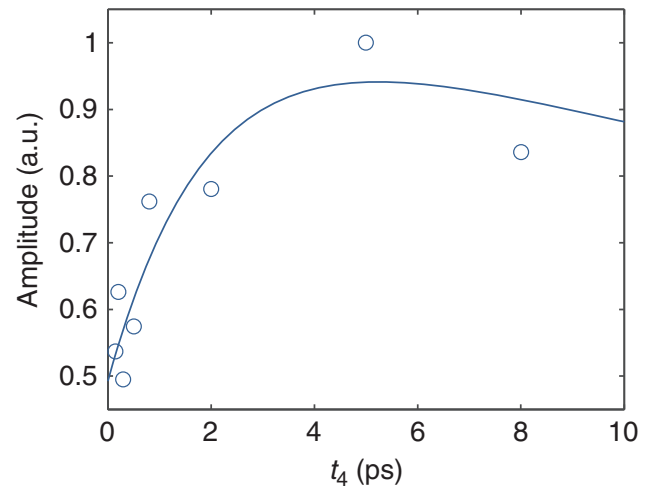

Figure 5 | Population time dependence of 3D cross peak amplitude. Experimental values (blue circles) and simulation trace (blue line) of the $\mathrm{H}$ / $M / L$ cross peak amplitudes in the quasi-2D spectra $\left(\lambda_{1}=656 \mathrm{~nm}\right.$ slice; $\left.t_{2}=0.3 \mathrm{ps}\right)$ as a function of the second population time $t_{4}$.

\section{Methods}

Sample preparation. The LHCII trimers sample was purified from spinach thylakoid membranes and solubilized with $\alpha$-dodecyl maltoside $(\alpha$-DM) using sucrose-gradient ultracentrifugation ${ }^{37}$. The sample was mixed with nitrogenflushed $0.06 \% \alpha$-DM and $10 \mathrm{mM}$ HEPES buffer solution, and placed in a $1 \mathrm{~mm}$ sapphire cell at room temperature. The absorbance of the sample at $674 \mathrm{~nm}$ was measured to be 0.2 . All linear, $2 \mathrm{D}$ and $3 \mathrm{D}$ spectra were acquired at room temperature.

Data acquisition. Our implementation of third-order $2 \mathrm{DES}^{38}$ and fifth-order $3 \mathrm{DES}^{17,27}$ has been described in detail elsewhere. In brief, in the pump-probe geometry ${ }^{39-42}$ implemented 3DES, the pulse shaper was used to create a four-pulse 'pump' train $(1 \mathrm{kHz}$ laser pulses from an optical parametric amplifier centred at $668 \mathrm{~nm}$. Pulse duration is $43 \mathrm{fs}$; see Fig. 2a) while a white light continuum pulse is used as the 'probe' (that provides the fifth interaction). In the present setup, the pulse shaper is able to produce pulse trains whereby the maximum delay between the first and fourth pulses is about 1 ps. Both coherence times $t_{1}$ and $t_{3}$ were increased in $7 \mathrm{fs}$ time steps from $0 \mathrm{fs}$ to $140 \mathrm{fs}$. The maximum population time $t_{2}$ for our present setup is therefore about $600 \mathrm{fs}$. The delay for the other population time $t_{4}$ is achieved by a conventional optomechanical delay stage, and the maximum achievable $t_{4}$ is determined by the length of the delay stage. To obtain the signal from the desired processes (Fig. 1b), phase cycling ${ }^{43-45}$ is necessary. A $3 \times 3 \times 3 \times 1$ phase cycling scheme $e^{27,45}$ was used to obtain the purely absorptive $3 \mathrm{D}$ spectra. For the experiments reported in this article, the population time $t_{2}$ was set at $300 \mathrm{fs}$, and population times $t_{4}$ were measured at $150,200,300,500,800$, $2,000,5,000$ and $8,000 \mathrm{fs}$. The $3 \mathrm{D}$ spectra were normalized to the maximum intensity of the $2 \mathrm{D}$ projection at frequency axis $\left(\lambda_{1}, \lambda_{3}\right)$. The pulse shaper was referenced to a carrier frequency of $420 \mathrm{THz}$ and the signal was collected in a partially rotating frame. The 'probe' beam is measured with a spectrometer (Acton SP2300, Princeton Instruments) and recorded with a 100-pixel $\times 1,340$-pixel charge-coupled device camera (PIXIS 100B, Princeton Instruments) giving the wavelength axis $\lambda_{5}$. The data is Fourier transformed about $t_{1}$ and $t_{3}$ to give the frequency axes $\omega_{1}$ and $\omega_{3}$, which was then converted to wavelength axes $\lambda_{1}$ and $\lambda_{3}$. Scatter subtraction and Fourier transformation into the frequency spectrum was performed as reported previously ${ }^{27,38}$. For the accompanying $2 \mathrm{DES}$, a two-pulse 'pump' train with a $3 \times 1$ phase cycling scheme ${ }^{38}$ is used. The population times $t_{2}$ were recorded at a series of delays from $120 \mathrm{fs}$ to $10,000 \mathrm{fs}$. In 2D optical spectroscopy, a common method to characterize possible experimental artefacts is to apply the $2 \mathrm{D}$ projection-slice theorem ${ }^{39}$. In this method, the $2 \mathrm{D}$ spectrum is integrated over one frequency axis and compared with the transient absorption spectrum. The $2 \mathrm{D}$ projection-slice theorem states that the $2 \mathrm{D}$ spectrum should be similar to the $1 \mathrm{D}$ spectrum. Analogous to the $2 \mathrm{D}$ counterpart, the $3 \mathrm{D}$ projectionslice theorem has also been developed ${ }^{13}$. We compare the experimental 3D spectra integrated along one of the frequency axes with the corresponding experimental 2D spectra to verify that our $3 \mathrm{D}$ spectra are largely free of experimental artefacts. The details of the comparison are provided in the Supplementary Note 1.

\section{References}

1. Jonas, D. M. Two-dimensional femtosecond spectroscopy. Annu. Rev. Phys. Chem. 54, 425-463 (2003).

2. Cho, M. Coherent two-dimensional optical spectroscopy. Chem. Rev. 108, 1331-1418 (2008).

3. Mukamel, S., Tanimura, Y. \& Hamm, P. Coherent multidimensional optical spectroscopy. Acc. Chem. Res. 42, 1207-1209 (2009).
4. Brixner, T. et al. Two-dimensional spectroscopy of electronic couplings in photosynthesis. Nature 434, 625-628 (2005).

5. Engel, G. S. et al. Evidence for wavelike energy transfer through quantum coherence in photosynthetic systems. Nature 446, 782-786 (2007).

6. Collini, E. et al. Coherently wired light-harvesting in photosynthetic marine algae at ambient temperature. Nature 463, 644-U669 (2010).

7. Schlau-Cohen, G. S., Ishizaki, A. \& Fleming, G. R. Two-dimensional electronic spectroscopy and photosynthesis: fundamentals and applications to photosynthetic light-harvesting. Chem. Phys. 386, 1-22 (2011).

8. Schlau-Cohen, G. S. et al. Pathways of energy flow in LHCII from twodimensional electronic spectroscopy. J. Phys. Chem. B 113, 15352-15363 (2009).

9. Novoderezhkin, V. I., Palacios, M. A., van Amerongen, H. \& van Grondelle, R. Energy-transfer dynamics in the LHCII complex of higher plants: modified redfield approach. J. Phys. Chem. B 108, 10363-10375 (2004).

10. Novoderezhkin, V. I., Palacios, M. A., Van Amerongen, H. \& Van Grondelle, R. Excitation dynamics in the LHCII complex of higher plants: modeling based on the 2.72 a crystal structure. J. Phys. Chem. B 109, 10493-10504 (2005).

11. Novoderezhkin, V., Marin, A. \& van Grondelle, R. Intra- and inter-monomeric transfers in the light harvesting LHCII complex: the Redfield-Förster picture. Phys. Chem. Chem. Phys. 13, 17093 (2011).

12. Renger, T., Madjet, M. E., Knorr, A. \& Muh, F. How the molecular structure determines the flow of excitation energy in plant light-harvesting complex II. J. Plant. Physiol. 168, 1497-1509 (2011).

13. Hamm, P. Three-dimensional-IR spectroscopy: beyond the two-point frequency fluctuation correlation function. J. Chem. Phys. 124, 124506 (2006)

14. Ding, F. \& Zanni, M. T. Heterodyned 3D IR spectroscopy. Chem. Phys. 341, 95-105 (2007).

15. Garrett-Roe, S. \& Hamm, P. What can we learn from three-dimensional infrared spectroscopy? Acc. Chem. Res. 42, 1412-1422 (2009).

16. Fidler, A. F., Harel, E. \& Engel, G. S. Dissecting hidden couplings using fifth-order three-dimensional electronic spectroscopy. J. Phys. Chem. Lett. 1, 2876-2880 (2010).

17. Zhang, Z., Wells, K. L. \& Tan, H. S. Purely absorptive fifth-order threedimensional electronic spectroscopy. Opt. Lett. 37, 5058-5060 (2012).

18. Mukherjee, S. S., Skoff, D. R., Middleton, C. T. \& Zanni, M. T. Fully absorptive 3D IR spectroscopy using a dual mid-infrared pulse shaper. J. Chem. Phys. 139, 144205 (2013).

19. Fulmer, E. C., Ding, F. \& Zanni, M. T. Heterodyned fifth-order 2D-IR spectroscopy of the azide ion in an ionic glass. J. Chem. Phys. 122, 1-12 (2005)

20. Borek, J. A., Perakis, F. \& Hamm, P. Testing for memory-free spectroscopic coordinates by 3D IR exchange spectroscopy. Proc. Natl Acad. Sci. USA 111, 10462-10467 (2014).

21. Dijkstra, A. G. \& Tanimura, Y. Linear and third- and fifth-order nonlinear spectroscopies of a charge transfer system coupled to an underdamped vibration. J. Chem. Phys. 142, 212423 (2015).

22. Turner, D. B., Stone, K. W., Gundogdu, K. \& Nelson, K. A. Three-dimensional electronic spectroscopy of excitons in GaAs quantum wells. J. Chem. Phys. 131, 144510 (2009).

23. Li, H., Bristow, A. D., Siemens, M. E., Moody, G. \& Cundiff, S. T. Unraveling quantum pathways using optical 3D Fourier-transform spectroscopy. Nat. Commun. 4, 1390 (2013).

24. Papagiannakis, E. et al. Use of ultrafast dispersed pump-dump-probe and pump-repump-probe spectroscopies to explore the light-induced dynamics of peridinin in solution. J. Phys. Chem. B 110, 512-521 (2006).

25. van Veldhoven, E., Khurmi, C., Zhang, X. Z. \& Berg, M. A. Time-resolved optical Spectroscopy with multiple population dimensions: a general method for resolving dynamic heterogeneity. Chemphyschem. 8, 1761-1765 (2007).

26. Blank, D. A., Kaufman, L. J. \& Fleming, G. R. Fifth-order two-dimensional Raman spectra of $\mathrm{CS}_{2}$ are dominated by third-order cascades. J. Chem. Phys. 111, 3105-3114 (1999).

27. Zhang, Z., Wells, K. L., Seidel, M. T. \& Tan, H. S. Fifth-order three-dimensional electronic spectroscopy using a pump-probe configuration. J. Phys. Chem. B 117, 15369-15385 (2013).

28. Gelin, M. F. \& Domcke, W. Simple recipes for separating excited-state absorption and cascading signals by polarization-sensitive measurements. J. Phys. Chem. A 117, 11509-11513 (2013).

29. Garrett-Roe, S. \& Hamm, P. Purely absorptive three-dimensional infrared spectroscopy. J. Chem. Phys. 130, 164510 (2009).

30. van Amerongen, H. \& Croce, R. Light harvesting in photosystem II. Photosynth. Res. 116, 251-263 (2013).

31. Pan, X. W., Liu, Z. F., Li, M. \& Chang, W. R. Architecture and function of plant light-harvesting complexes II. Curr. Opin. Struct. Biol. 23, 515-525 (2013).

32. Mukamel, S. Principles of Nonlinear Optical Spectroscopy (Oxford University Press, 1995). 
33. Liu, Z. F. et al. Crystal structure of spinach major light-harvesting complex at 2.72 angstrom resolution. Nature 428, 287-292 (2004).

34. Wells, K. L., Lambrev, P. H., Zhang, Z., Garab, G. \& Tan, H.-S. Pathways of energy transfer in LHCII revealed by room-temperature $2 \mathrm{D}$ electronic spectroscopy. Phys. Chem. Chem. Phys. 16, 11640-11646 (2014).

35. Linnanto, J. et al. Excitation energy transfer in the LHC-II trimer: a model based on the new 2.72 angstrom structure. Photosynth. Res. 87, 267-279 (2006).

36. Muh, F., Madjet, M. E. A. \& Renger, T. Structure-based identification of energy sinks in plant light-harvesting complex II. J. Phys. Chem. B 114, 13517-13535 (2010).

37. Caffarri, S., Croce, R., Breton, J. \& Bassi, R. The major antenna complex of photosystem II has a xanthophyll binding site not involved in light harvesting. J. Biol. Chem. 276, 35924-35933 (2001).

38. Zhang, Z., Wells, K. L., Hyland, E. W. J. \& Tan, H.-S. Phase-cycling schemes for pump-probe beam geometry two-dimensional electronic spectroscopy. Chem. Phys. Lett. 550, 156-161 (2012).

39. Faeder, S. M. G. \& Jonas, D. M. Two-dimensional electronic correlation and relaxation spectra: theory and model calculations. J. Phys. Chem. A 103, 10489-10505 (1999).

40. Shim, S. H., Strasfeld, D. B., Ling, Y. L. \& Zanni, M. T. Automated 2D IR spectroscopy using a mid-IR pulse shaper and application of this technology to the human islet amyloid polypeptide. Proc. Natl Acad. Sci. USA 104, 14197-14202 (2007).

41. DeFlores, L. P., Nicodemus, R. A. \& Tokmakoff, A. Two-dimensional Fourier transform spectroscopy in the pump-probe geometry. Opt. Lett. 32, 2966-2968 (2007).

42. Myers, J. A., Lewis, K. L. M., Tekavec, P. F. \& Ogilvie, J. P. Two-color twodimensional Fourier transform electronic spectroscopy with a pulse-shaper. Opt. Express 16, 17420-17428 (2008).

43. Keusters, D., Tan, H. S. \& Warren, W. S. Role of pulse phase and direction in twodimensional optical spectroscopy. J. Phys. Chem. A 103, 10369-10380 (1999).

44. Tian, P., Keusters, D., Suzaki, Y. \& Warren, W. S. Femtosecond phase-coherent two-dimensional spectroscopy. Science 300, 1553-1555 (2003).

45. Tan, H.-S. Theory and phase-cycling scheme selection principles of collinear phase coherent multi-dimensional optical spectroscopy. J. Chem. Phys. 129, 124501 (2008).

\section{Acknowledgements}

We thank J.R. Rouxel for discussions. This work is supported by a joint grant from the Singapore Agency for Science, Technology and Research, A*STAR and the Hungarian National Innovation Office (A ${ }^{\star}$ STAR SERC Grant No. 102-149-0153; NIH-A ${ }^{\star}$ STAR

TET_10-1-2011-027), the Singapore National Research Foundation (NRF-CRP5-2009-04) the Hungarian Scientific Research Fund (OTKA-PD 104530 to P.H.L.) and the European Commission (TÁMOP 4.2.2.D-15/1 / KONV-2015-0024 and OTKA-K112688 to G.G.). Z.Z. thanks the Nanyang President's Graduate Scholarship for support.

\section{Author contributions}

Z.Z. and H.-S.T. designed the experiment. P.H.L. isolated and purified the LHCII sample. Z.Z. and K.L.W. prepared the sample and optical setup for measurement and Z.Z. collected the data; Z.Z., P.H.L. and H.-S.T. performed the data analysis. Z.Z., P.H.L. and H.-S.T. wrote the paper, and all authors discussed the results and commented on the manuscript

\section{Additional information}

Supplementary Information accompanies this paper at http://www.nature.com/ naturecommunications

Competing financial interests: The authors declare no competing financial interests.

Reprints and permission information is available online at http://npg.nature.com/ reprintsandpermissions/

How to cite this article: Zhang, Z. et al. Direct observation of multistep energy transfer in LHCII with fifth-order 3D electronic spectroscopy. Nat. Commun. 6:7914 doi: $10.1038 /$ ncomms8914 (2015)

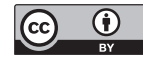

This work is licensed under a Creative Commons Attribution 4.0 International License. The images or other third party material in this article are included in the article's Creative Commons license, unless indicated otherwise in the credit line; if the material is not included under the Creative Commons license, users will need to obtain permission from the license holder to reproduce the material. To view a copy of this license, visit http://creativecommons.org/licenses/by/4.0/ 